\title{
Origin of low thermal conductivity in In4Se3
}

Article

Accepted Version

Luu, S. D. N., Supka, A. R., Nguyen, V. H., Vo, D.-V., Hung, N., Wojciechowski, K. T., Fornari, M. and Vaqueiro, P. ORCID: https://orcid.org/0000-0001-7545-6262 (2020) Origin of low thermal conductivity in In4Se3. ACS Applied Energy Materials, 3 (12). pp. 12549-12556. ISSN 2574-0962 doi: https://doi.org/10.1021/acsaem.0c02489 Available at https://centaur.reading.ac.uk/94436/

It is advisable to refer to the publisher's version if you intend to cite from the work. See Guidance on citing.

To link to this article DOI: http://dx.doi.org/10.1021/acsaem.0c02489

Publisher: ACS Publications

All outputs in CentAUR are protected by Intellectual Property Rights law, including copyright law. Copyright and IPR is retained by the creators or other copyright holders. Terms and conditions for use of this material are defined in the End User Agreement.

\section{www.reading.ac.uk/centaur}

\section{CentAUR}


Central Archive at the University of Reading

Reading's research outputs online 
This document is confidential and is proprietary to the American Chemical Society and its authors. Do not copy or disclose without written permission. If you have received this item in error, notify the sender and delete all copies.

\section{Origin of Low Thermal Conductivity in In4Se3}

\begin{tabular}{|r|l|}
\hline Journal: & ACS Applied Energy Materials \\
\hline Manuscript ID & ae-2020-02489q.R1 \\
\hline Manuscript Type: & Article \\
\hline Aute Submitted by the & 13-Nov-2020 \\
\hline Complete List of Authors: & $\begin{array}{l}\text { Luu, Son D N; Institute of Research and Development, Duy Tan } \\
\text { Univeristy } \\
\text { Supka, Andrew ; Department of Physics and Science of Advanced } \\
\text { Materials Program } \\
\text { Nguyen, Van Huy ; Key Laboratory of Advanced Materials for Energy and } \\
\text { Environmental Applications } \\
\text { Vo, Dai-Viet N. ; Center of Excellence for Green Energy and } \\
\text { Environmental Nanomaterials (CE@GrEEN) } \\
\text { Tuan Hung, Nguyen; Tohoku University, Frontier Research Institute for } \\
\text { Interdisciplinary Sciences } \\
\text { Wojciechowski, Krzystof; AGH University of Science and Technology, } \\
\text { faculty of Materials Science and Ceramics } \\
\text { Fornari, Marco; Central Michigan University, Physics } \\
\text { Vaqueiro, Paz; University of Reading, Chemistry }\end{array}$ \\
\hline
\end{tabular}




\section{Origin of Low Thermal Conductivity in $\mathrm{In}_{4} \mathrm{Se}_{3}$}

Son D. N. Luu ${ }^{1 *}$, Andrew R. Supka², Van Huy Nguyen³, Dai-Viet N. Vo', Nguyen T. Hung, Krzysztof T. Wojciechowski6,7, Marco Fornari2, Paz Vaqueiro ${ }^{*}$

${ }^{1}$ Institute of Research and Development, Duy Tan University, Da Nang, 550000, Viet Nam

${ }^{2}$ Department of Physics and Science of Advanced Materials Program, Central Michigan University, Mt. Pleasant, Michigan, 48859 USA

${ }^{3}$ Key Laboratory of Advanced Materials for Energy and Environmental Applications, Lac Hong University, Dong Nai 810000, Viet Nam

${ }^{4}$ Center of Excellence for Green Energy and Environmental Nanomaterials (CE@GrEEN), Nguyen Tat Thanh University, 300A Nguyen Tat Thanh, District 4, Ho Chi Minh City 755414, Viet Nam

${ }^{5}$ Frontier Research Institute for Interdisciplinary Sciences, Tohoku University, Sendai, 9808578, Japan

${ }^{6}$ AGH University of Science and Technology, Faculty of Materials Science and Ceramics, Thermoelectric Research Laboratory, 30 Mickiewicza, 30-059 Cracow, Poland

${ }^{7}$ The Lukasiewicz Research Network -, The Institute of Advanced Manufacturing Technology, Centre of Thermoelectric Materials Research, 37A Wroclawska, 30-011 Cracow, Poland ${ }^{8}$ Department of Chemistry, University of Reading, Whiteknights Park, Reading RG6 6AD, England, United Kingdom 


\section{Abstract}

$\ln _{4} \mathrm{Se}_{3}$ is an attractive $n$-type thermoelectric material for mid-range waste heat recovery, owing to its low thermal conductivity $\left(\sim 0.9 \mathrm{~W} \cdot \mathrm{m}^{-1} \cdot \mathrm{K}^{-1}\right.$ at $\left.300 \mathrm{~K}\right)$. Here, we explore the relationship between the elastic properties, thermal conductivity and structure of $\ln _{4} \mathrm{Se}_{3}$. The experimentallydetermined average sound velocity $\left(2010 \mathrm{~m} \mathrm{~s}^{-1}\right)$, Young's modulus (47 GPa), and Debye temperature $(198 \mathrm{~K})$ of $\ln _{4} \mathrm{Se}_{3}$ are rather low, indicating considerable lattice softening. This behavior, which is consistent with low thermal conductivity, can be related to the complex bonding found in this material, in which strong covalent In-In and In-Se bonds coexist with weaker electrostatic interactions. Phonon dispersion calculations show that Einstein-like modes occur at $\approx 30 \mathrm{~cm}^{-1}$. These Einstein-like modes can be ascribed to weakly bonded $\mathrm{In}^{+}$cations located between strongly-bonded $\left[\left(\ln _{3}\right)^{5+}\left(\mathrm{Se}^{2-}\right)_{3}\right]^{-}$layers. The Grüneisen parameter for the softbonded $\mathrm{In}^{+}$at the frequencies of the Einstein-like modes is large, indicating a high degree of bond anharmonicity and hence increased phonon scattering. The calculated thermal conductivity and elastic properties are in good agreement with experimental results. 


\section{Keywords}

Thermoelectric materials, Thermal conductivity, Grüneisen parameter, Lattice softening, Lone

pair. 


\section{INTRODUCTION}

Worldwide concerns with energy supply and sustainability have stimulated considerable research efforts into thermoelectric materials, which enable direct conversion of waste heat into electrical power. The efficiency of thermoelectric energy recovery is related to the dimensionless thermoelectric figure of merit, $\mathrm{ZT}$, which is given by $\mathrm{ZT}=\mathrm{S}^{2} \sigma \mathrm{T} /\left(\kappa_{\mathrm{L}}+\kappa_{\mathrm{e}}\right)$ where $\mathrm{S}, \sigma, \mathrm{T}, \kappa_{\mathrm{L}}$, and $\kappa_{\mathrm{e}}$ are the Seebeck coefficient, electrical conductivity, absolute temperature, lattice, and electronic thermal conductivities, respectively ${ }^{1}$. To maximize $\mathrm{ZT}$, materials with low thermal conductivity are required. As a consequence of Wiedemann-Franz law, reducing the electronic thermal conductivity, $\kappa_{\mathrm{e}}$, would simultaneously lower the electrical conductivity, $\sigma$. Therefore, strategies to reduce the thermal conductivity focus on the lattice component $\left(\kappa_{\mathrm{L}}\right)$, which is related to vibrational energy transport. These strategies include the introduction of species with low-energy

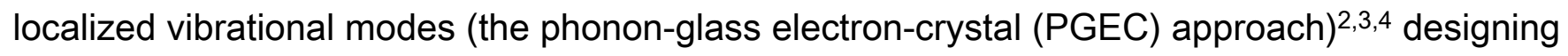
materials with part-crystalline part-liquid states (the phonon-liquid electron-crystal (PLEC) approach $)^{4,5,6,7,8}$, grain-boundary engineering ${ }^{9,10}$, and the introduction of nano-inclusions ${ }^{10,11}$. 
Understanding the origin of the intrinsically low lattice thermal conductivity found in some thermoelectric materials is critically important to facilitate the discovery of the next generation of high-performance candidates ${ }^{12,16}$. Pseudo-layered $\ln _{4} \mathrm{Se}_{3}$ (Figure 1), a mixed-valence compound that can be formulated as $\left(\ln ^{+}\right)\left[\left(\ln _{3}\right)^{5+}\left(\mathrm{Se}^{2-}\right)_{3}\right]^{-}$, is one of the best performing $n$-type thermoelectric materials for mid-range waste heat recovery ${ }^{17,32}$. The thermoelectric properties of $\ln _{4} \mathrm{Se}_{3}$ are highly anisotropic due to its pseudo-layered structure. Single crystals of $\operatorname{In}_{4} \mathrm{Se}_{3-\delta}(\delta$ $=0.65$ ) exhibit an impressive $\mathrm{ZT} \approx 1.48$ at $705 \mathrm{~K}$ in the direction parallel to the layers, but a much lower $\mathrm{ZT},<0.5$, perpendicular to the layers ${ }^{17}$. It has been reported that multiple doping is an effective strategy to produce polycrystalline samples with similarly high values of $Z T$, as exemplified by $\mathrm{Pb} / \mathrm{Sn}$-co-doped $\ln _{4} \mathrm{Se}_{3}{ }^{19}(\mathrm{ZT}=1.4$ at $733 \mathrm{~K})$. The outstanding thermoelectric performance of $\ln _{4} \mathrm{Se}_{3}$ has been attributed to its low thermal conductivity, which is $\sim 0.9 \mathrm{~W} \cdot \mathrm{m}^{-}$ $1 \cdot \mathrm{K}^{-1}$ for the undoped polycrystalline material at room temperature ${ }^{17,18,19,32}$, while in doped and selenium-deficient samples, values as low as $\sim 0.4 \mathrm{~W} \cdot \mathrm{m}^{-1} \cdot \mathrm{K}^{-1}$ at $723 \mathrm{~K}$ can be reached ${ }^{30,31}$. 
(a)

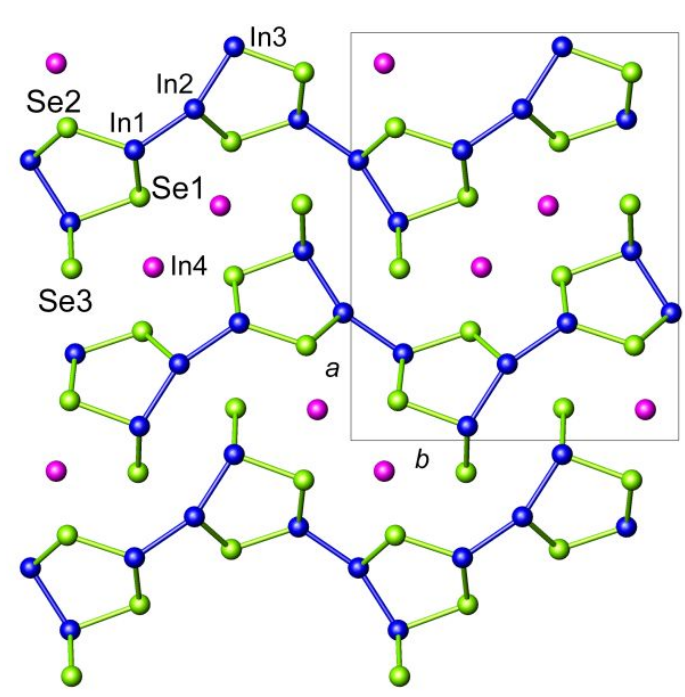

(b)

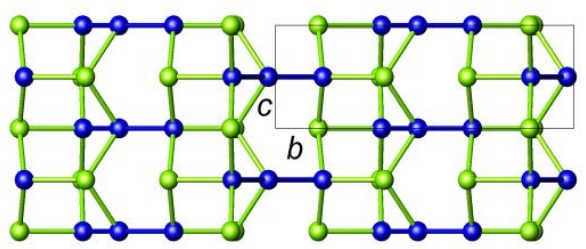

Figure 1. (a) View of the crystal structure of $\ln _{4} \mathrm{Se}_{3}$ along [001]. The $\ln 1, \ln 2, \ln 3$ atoms (dark blue spheres) form $\left(\ln _{3}\right)^{5+}$ clusters and are covalently bonded to the selenium atoms (green spheres). The In4 atoms (dark pink spheres) are located between the $\left[\left(\ln _{3}\right)^{5+}\left(\mathrm{Se}^{2-}\right)_{3}\right]^{-}$layers. $(\mathrm{b})$ View of a $\left[\left(\mathrm{In}_{3}\right)^{5+}\left(\mathrm{Se}^{2-}\right)_{3}\right]^{-}$layer along [100]. The unit cell is shown as a grey rectangle.

The low thermal conductivity of selenium-deficient $\ln _{4} \mathrm{Se}_{3-\delta}$ has been proposed that is the result of charge density wave (CDW) induced by a quasi-one-dimensional lattice Peierls distortion ${ }^{17}$. This, however, has been questioned by Osters and co-workers ${ }^{33}$, who found that $\ln _{4} \mathrm{Se}_{3}$ behaves as a line phase and does not accommodate selenium deficiency. Instead, selenium-deficient 


\begin{abstract}
samples were found to contain indium metal ${ }^{32}$, while single-crystal X-ray diffraction data provide no evidence of a $C D W^{33}$. Moreover, given that stoichiometric $\ln _{4} \mathrm{Se}_{3}$ already exhibits an unusually low thermal conductivity, the investigation of the origin of the low thermal conductivity of this material is essential.
\end{abstract}

There is a strong link between the elastic properties and the lattice thermal conductivity of a given material ${ }^{34}$, but little is known about the elastic properties of $\ln _{4} \mathrm{Se}_{3}{ }^{35,36}$. Here we describe the correlation between structure and elastic and thermal properties of polycrystalline $\ln _{4} \mathrm{Se}_{3}$. With the aid of first-principles calculations, we explore the interplay between bonding, phonon dispersions, and mechanical properties in this material. Our results demonstrate that soft bonding of $\mathrm{In}^{+}$ions in the pseudo-layered structure of $\operatorname{In}_{4} \mathrm{Se}_{3}$ is key to interpret the root of low thermal conductivity in this material.

\title{
EXPERIMENTAL
}

\section{Synthesis and structural characterization}

The synthetic procedure for the preparation of $\ln _{4} \mathrm{Se}_{3}$ and the Rietveld refinement using powder X-ray diffraction data were presented in previous work ${ }^{32}$. Powder X-ray diffraction data 
for the powder and the pellet have been included as Supporting Information (SI, Figure S1).

Significant bond lengths and angles are included in the SI (Table S1\&2). SEM and EDS measurements are consistent with the nominal composition of $\ln _{4} \mathrm{Se}_{3}(\mathrm{SI}$, Table S3).

\section{Property measurements}

A pellet (density >95\%) with a diameter of $10 \mathrm{~mm}$ and a thickness of $\sim 2.47 \mathrm{~mm}$ was used to measure the longitudinal and transverse acoustic velocities using an ultrasonic instrument Panametrics Epoch III. Details of this measurement technique are given elsewhere ${ }^{37}$. These measured velocities were used to calculate the elastic parameters, and the Poisson ratio ${ }^{38}$. The average sound velocity of the sample was calculated from the longitudinal $\left(v_{l}\right)$ and the transverse $\left(v_{t}\right)$ sound velocities using the following expression ${ }^{39,40:}$

$$
v_{a}=\left(\frac{1}{3}\left[\frac{1}{v_{l}^{3}}+\frac{2}{v_{t}^{3}}\right]\right)^{-1 / 3}
$$

These values were also used to calculate the Poisson ratio (up) using the following relationship ${ }^{41}$ :

$$
\mathrm{v} p=\frac{1-2\left({ }^{(} t / v_{l}\right)^{2}}{2-2\left({ }^{v} t / v_{l}\right)^{2}}
$$

The elastic $\left(\gamma_{e}\right)$ parameter, and Young's modulus $(\mathrm{E})$ were calculated using the equations ${ }^{42}$ : 


$$
\begin{array}{r}
\gamma_{e}=\frac{3}{2}\left(\frac{1+v p}{2-3 v p}\right) \\
E=\frac{\rho v_{s}^{2}\left(3 v_{l}^{2}-4 v_{t}^{2}\right)}{\left(v_{l}^{2}-v_{t}^{2}\right)}
\end{array}
$$

where $\rho$ is the density of the material. To estimate the Debye temperature, $\theta_{D}$, the average sound velocity was used in the expression ${ }^{39}$ :

$$
\theta_{D}=\frac{h}{k_{B}}\left(\frac{3 N}{4 \pi V}\right)^{-1 / 3} v_{a}(5)
$$

where $\mathrm{V}$ is the unit-cell volume; $\mathrm{N}$ is the number of atoms in a unit cell; $\mathrm{k}_{\mathrm{B}}$ is the Boltzmann constant, and $h$ is the Plank constant.

The electrical and thermal conductivities were measured and presented in ref ${ }^{32}$. The electronic $\left(\kappa_{\mathrm{e}}\right)$ and lattice $\left(\kappa_{\text {lat }}\right)$ thermal conductivities were estimated using the electrical conductivity data ${ }^{32}$ in conjunction with the Wiedemann-Franz law:

$$
\kappa_{\mathrm{e}}=\mathrm{L} \sigma \mathrm{T}
$$

where $\sigma$ is the electrical conductivity and $\mathrm{L}$ is the Lorenz number. The value of the Lorenz number ${ }^{43}$ was estimated using the expression $\mathrm{L}=1.5+\exp [-|\mathrm{S}| / 116]$, where $\mathrm{L}$ is in $10^{-8} \mathrm{~W} \Omega \mathrm{K}^{-2}$ and $\mathrm{S}$ in $\mu \mathrm{V} \mathrm{K}-1$ The minimum lattice thermal conductivity $\kappa_{\text {lat, min }}$ of $\ln _{4} \mathrm{Se}_{3}$ was estimated taking into account that ${ }^{44}$ :

$$
\kappa_{\text {lat }}=\frac{1}{3} \mathrm{C}_{\mathrm{v}} v_{\mathrm{a}} \Lambda
$$


(where $\mathrm{C}_{\mathrm{v}}$ and $\Lambda$ are the volumetric isochoric heat capacity and the phonon mean free path),

by using the interatomic distance as the minimum phonon mean free path. $\kappa_{\text {lat, } \min }$ was also estimated at a high temperature limit using Cahill's model ${ }^{14,45}$ :

$$
\kappa_{\min }=\frac{1}{2}\left(\frac{\pi}{6}\right)^{1 / 3} k_{B} V^{-2 / 3}\left(v_{l}+2 v_{t}\right)
$$

\section{First principle calculations}

Band structure, density of states, and phonon dispersions were computed using the Quantum

EXPRESSO package ${ }^{46}$ as integrated in AFLOW $\pi^{47}$. The Perdew-Burke-Ernzerhof (PBE)

functional was used to describe the exchange-correlation potential. Optimized norm-conserving

PBE pseudopotentials ${ }^{48}$, with a well-converged basis, set corresponding to an energy cut-off of 80 Ry, were used for the wavefunctions. To integrate over the Brillouin zone, a $2 \times 4 \times 8$ (shifted) grid was used. Electronic transport coefficients were evaluated with PAOFLOW ${ }^{49}$. The finite difference method using a $1 \times 2 \times 4$ supercell was employed to compute phonons. AFLOWm uses ElaStic ${ }^{50}$ to determine the nine independent elastic constants, $C_{i j}$, of orthorhombic crystals with Pnnm space group. The Young modulus and the Poisson ratio were calculated based on 
the $C_{i j}$, by using the Voigt, Reuss, and Hill equations of state. The mode resolved Grüneisen parameters were computed within the quasi-harmonic approximation and the lattice thermal conductivity was estimated using the Debye-Callaway model ${ }^{51}$.

\section{RESULTS AND DISCUSSION}

\section{Structure and bonding}

$\operatorname{In}_{4} \mathrm{Se}_{3}$ can be formulated as $\left(\mathrm{In}^{+}\right)\left[\left(\mathrm{In}_{3}\right)^{5+}\left(\mathrm{Se}^{2-}\right)_{3}\right]^{-}$, indicating the coexistence of covalent and ionic bonding ${ }^{52}$. The crystal structure of $\ln _{4} \mathrm{Se}_{3}$ (Figure 1) contains anionic layers, perpendicular to the a-axis, with stoichiometry $\left[\left(\mathrm{In}_{3}\right)^{5+}\left(\mathrm{Se}^{2-}\right)_{3}\right]^{-}$. These layers consist of interlocked pentameric $\mathrm{In}_{3} \mathrm{Se}_{2}$ rings, oriented along the c-axis, and linked into bulked layers by linear $\left(\ln _{3}\right)^{5+}$ cations. Within the $\left(\ln _{3}\right)^{5+}$ cluster, the distance between $\ln 1$ and $\ln 2$ atoms (refer to Figure 1 for atom labels) is $2.7239(7) \AA$ while the distance between $\ln 2$ and $\ln 3$ is $2.7703(6) \AA$. These values are well below those found in indium metal $(3.252 \text { and } 3.377 \AA)^{53}$, and are comparable to the sum of the covalent radius for two indium atoms, which is $2.88 \AA$. Within this layer, the In-Se bond distances (SI, Table S1) are also close to the sum of covalent radii for indium (1.44 $\AA$ ) and selenium $(1.20 \AA)^{54}$. This indicates that strong covalent bonding occurs within the $\left[\left(\ln _{3}\right)^{5+}\left(\mathrm{Se}^{2-}\right)_{3}\right]^{-}$ 
layers. Assuming tetrahedral coordination for the selenium atoms, Se3 exhibits two In-Se bonds and two dangling bonds (unoccupied coordination sites) whilst Se1 and Se2 possess three InSe bonds one dangling bond (Figure 1).

(a)

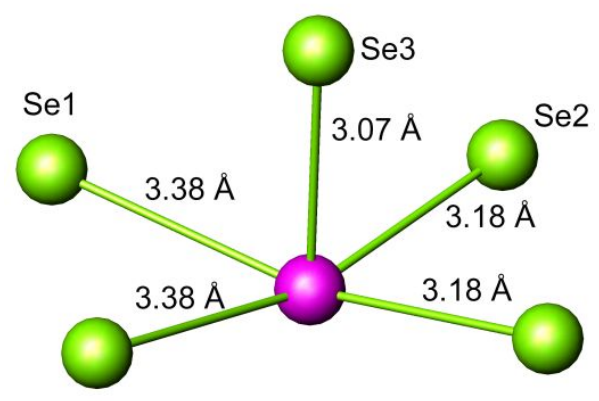

(b)

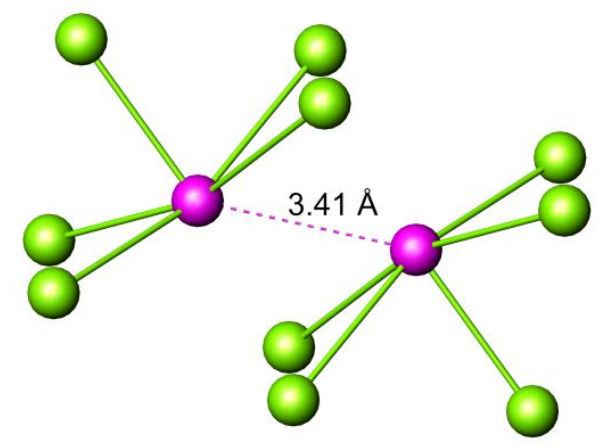

Figure 2. (a) Coordination environment of In4. (b) View of the $\ln 4-\ln 4$ interaction. Key: In4, dark pink spheres; selenium, green spheres.

Bond valence sums are consistent with a lower oxidation state for $\ln 4$ (SI, Table S2). This atom, which has a formal oxidation state of $\operatorname{In}^{+}$(electronic configuration $[\mathrm{Kr}] 5 s^{2}$ ), is located between the layers. The distance between In4 and the nearest indium atoms within the layers, In1 and In2, are 3.8379(7) $\AA$ and $3.7530(7)$, respectively, which are considerably larger than those in indium metal. The In4-Se distances are also considerably longer than those within the 
$\left[\left(\mathrm{In}_{3}\right)^{5+}\left(\mathrm{Se}^{2-}\right)_{3}\right]^{-}$layers. In4 adopts distorted square-pyramidal coordination (Figure 2(a)), with InSe distances ranging between 3.0688(1) and 3.3802(1) $\AA$ (SI, Table S1). These are close to the sum of ionic radii for $\operatorname{In}^{+}(1.32 \AA)^{55}$, and $\mathrm{Se}^{2-}(1.98 \AA)^{56}$. This suggests that $\mathrm{In}^{+}$cations are held between the layers by electrostatic interactions, while the $\left[\left(\ln _{3}\right)^{5+}\left(\mathrm{Se}^{2-}\right)_{3}\right]^{-}$layers are connected by strong and directional covalent bonds.
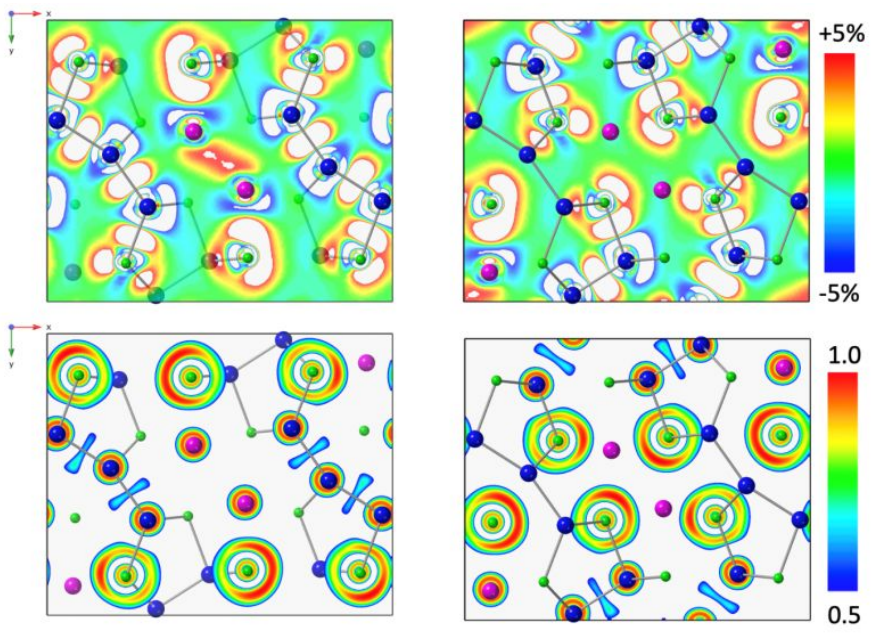

Figure 3. Charge density (top) and ELF (bottom) contour plots in the [001] planes crossing the $c$-axis at fractional coordinates of 0.0 (left) and 0.5 (right). The charge density color scale is centered on the mean value. Meaningful values of the ELF range from 0.5 to close to 1.0. Ions are colored as in Figure 1. 
The different nature of the bonding of In4 is reflected in its considerably larger atomic displacement parameter than those for the $\left(\ln _{3}\right)^{5+}$ cation found in the covalent layers, evident in single-crystal diffraction studies ${ }^{33}$. For instance, the atomic displacement parameter for $\ln 4$ found by Osters and coworkers ${ }^{33}$ is $60 \%$ larger than those in the $\left(\mathrm{In}_{3}\right)^{5+}$ cation.

The above considerations are entirely consistent with the results arising from first-principles electronic structure calculations. The band structure (SI, Figure S3) is in agreement with previously reported results ${ }^{57}$, with the density of states at the top of the valence band dominated by Se $p$ and In4 $s$ states. The presence of anti-bonding states with a substantial degree of cation $s$ character at the top of the valence band is a distinctive feature of semiconductors containing elements with lone pairs ${ }^{58}$, such as the $\mathrm{In}^{+}$cation present in $\ln _{4} \mathrm{Se}_{3}$. The electrical conductivity and the Seebeck coefficients computed as a function of the chemical potential from 300 to 700 $\mathrm{K}$ can be found in the SI (Figure S4).

Figure 3 shows contour plots of the valence charge density and the electron localization factors (ELF) in two [001] planes. The covalent nature of the bonding within the $\left[\left(\ln _{3}\right)^{5+}\left(\mathrm{Se}^{2-}\right)_{3}\right]^{-}$layers is reflected in the valence charge concentrated in the middle of the In-In and In-Se bonds within 
these layers, which is evident in these plots. The dangling bonds associated with the selenium atoms are also clearly observable, as asymmetrically localized electron clouds. By contrast, the nearly spherical ELF around In4 is consistent with ionic bonding. The square-pyramidal coordination of $\ln 4$ would be consistent with the presence of a lone pair of $5 s^{2}$ electrons at the missing octahedral vertex. Along the direction of this missing vertex, each $\ln 4$ atom has a neighboring In4 at a distance of $3.4082(3) \AA$ (Figure 2(b)). While this distance is larger than those in the $\left(\ln _{3}\right)^{5+}$ cluster, it is of the same order as those found in In metal. In the valence charge plot (Figure 3), there is evidence of charge concentrated between pairs of In 4 atoms, suggesting that these may be forming dimers.

\section{Thermal conductivity}

The heat capacity, thermal diffusivity, and total thermal conductivity of polycrystalline $\ln _{4} \mathrm{Se}_{3}$ as a function of temperature (Figure 4), previously presented $\mathrm{in}^{32}$, are in good agreement with previous reports ${ }^{28,29}$. The lattice thermal conductivity is the main contributor ( $\left.\kappa_{\text {latt }} \sim 99.0 \%\right)$ to the total thermal conductivity of $\ln _{4} \mathrm{Se}_{3}$ (Table 1). The temperature dependence of the thermal conductivity computed with the Debye-Callaway model (Figure 4) is in superb coincidence with 
the experimental values. By using the interatomic distance as the phonon mean free path $(\Lambda \sim$ $3.2 \AA$ ), we estimated that $\kappa_{\text {lat,min }}$ for $\ln _{4} \mathrm{Se}_{3}$ is $\sim 0.3 \mathrm{~W} \cdot \mathrm{m}^{-1} \cdot \mathrm{K}^{-1}$ at room temperature, while with Cahill's model, a value of $\kappa_{\text {lat,min }}$ of $\sim 0.4 \mathrm{~W} \cdot \mathrm{m}^{-1} \cdot \mathrm{K}^{-1}$ is found. Our experimental value of $\kappa_{\text {lat }}$ is $\sim$ $0.84 \mathrm{~W} \cdot \mathrm{m}^{-1} \cdot \mathrm{K}^{-1}$ at $323 \mathrm{~K}$ (Table 1 ), indicating that $\Lambda$ of $\mathrm{In}_{4} \mathrm{Se}_{3}$ is larger than the interatomic distance. Therefore, there is still potential for further reductions in thermal conductivity. Indeed, the incorporation of nano-inclusions in $\ln _{4} \mathrm{Se}_{3}{ }^{27}$ leads to values of thermal conductivity close to its minimum value.

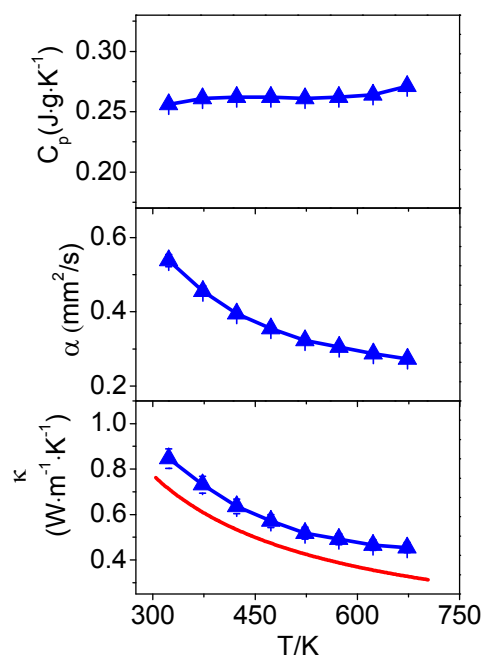

Figure 4. The specific heat, thermal diffusivity, and thermal conductivity of $\ln _{4} \mathrm{Se}_{3}$ as a function of temperature (blue triangles). The temperature dependence of the thermal conductivity 
computed with the Debye-Callaway model using parameters from the first-principles is shown as a red line.

Table 1. The electrical conductivity $(\sigma)$, electronic thermal $\left(\kappa_{\mathrm{e}}\right)$, lattice thermal $\left(\kappa_{\text {lat }}\right)$, and total thermal $\left(\kappa_{\text {tot }}\right)$ conductivities at $323 \mathrm{~K}$.

\begin{tabular}{lllll}
\hline & $\sigma(\mathrm{S} / \mathrm{m})$ & $\kappa_{\mathrm{e}}\left(\mathrm{W} \cdot \mathrm{m}^{-1} \cdot \mathrm{K}^{-1}\right)$ & $\kappa_{\text {latt }}\left(\mathrm{W} \cdot \mathrm{m}^{-1} \cdot \mathrm{K}^{-1}\right)$ & $\kappa_{\text {tot }}\left(\mathrm{W} \cdot \mathrm{m}^{-1} \cdot \mathrm{K}^{-1}\right)$ \\
\hline \hline $\mathrm{In}_{4} \mathrm{Se}_{3}$ & 1965 & 0.01 & 0.84 & 0.85
\end{tabular}

\section{Elastic properties}

The nine elastic constants calculated by us are consistent with the experimental results reported in the literature (Table 2). The elastic properties for $\ln _{4} \mathrm{Se}_{3}$ determined experimentally and through our first-principles calculations are summarized in Table 3. The experimentallydetermined sound velocities for $\ln _{4} \mathrm{Se}_{3}$, which in the Debye model would correspond to the group velocities of the heat-carrying acoustic phonons, are rather low. These velocities are reasonably consistent with the calculated values of the transverse sound velocities, 1381 and $1650 \mathrm{~m} \mathrm{~s}^{-1}$, and the longitudinal sound velocity, $2870 \mathrm{~m} \mathrm{~s}^{-1}$. Given that it has been shown that $\kappa_{\text {lat }}$ is directly 
proportional to the cube of the average sound velocity ${ }^{59}$, a low sound velocity will result in a low thermal conductivity. The Young's modulus of $\ln _{4} \mathrm{Se}_{3}(\mathrm{E} \sim 47 \mathrm{GPa})$, which is related to its stiffness (i.e. its chemical bond strength), is also low. For instance, the Young's modulus of $\ln _{4} \mathrm{Se}_{3}$ is significantly lower than those of established thermoelectric materials such as $\mathrm{Si}_{0.8} \mathrm{Ge}_{0.2}{ }^{60}(\mathrm{E} \sim 143 \mathrm{GPa})$ and $\mathrm{Mg}_{2} \mathrm{Si} 61(\mathrm{E} \sim 117 \mathrm{GPa})$, and comparable to other state-of-the art thermoelectric materials, including $\mathrm{SnSe}^{37}$ (E 28-40 GPa), $\mathrm{PbSe}^{37}$ (E 62-65 GPa), $\mathrm{PbTe}^{37,62}$ (E 54-57 GPa), $\mathrm{Cu}_{2} \mathrm{Se}^{63}(\mathrm{E} \sim 65-68 \mathrm{GPa})$ or those of glass and porous materials, such as borosilicate glass (E 61-64 GPa), brick (E 10-50 GPa) and concrete (E 25-38 GPa) ${ }^{64}$.

Table 2. Elastic constants for $\ln _{4} \mathrm{Se}_{3}$ in $\mathrm{GPa}$. The experimental data are from ref. ${ }^{36}$

\begin{tabular}{|l|l|l|l|l|l|l|l|l|l|}
\hline & $\mathrm{C}_{11}$ & $\mathrm{C}_{22}$ & $\mathrm{C}_{33}$ & $\mathrm{C}_{44}$ & $\mathrm{C}_{55}$ & $\mathrm{C}_{66}$ & $\mathrm{C}_{12}$ & $\mathrm{C}_{13}$ & $\mathrm{C}_{23}$ \\
\hline This study & 37.6 & 66.7 & 56.7 & 13.7 & 23.7 & 19.9 & 17.9 & 28.0 & 15.4 \\
\hline Experimental & 38.2 & 66.5 & 64.3 & 16.6 & 26.6 & 19.0 & 10.8 & 30.4 & 22.4 \\
\hline
\end{tabular}

Materials with weak interatomic bonding usually possess low stiffness and Young's modulus.

They are regarded as "softly" bonded materials that result in flattened phonon dispersion curves, and therefore, low sound velocities and low thermal conductivities ${ }^{65}$. Theoretically, the value of 
Young's modulus is computed assuming a specific equation of state (EoS), and the calculated values using the Voigt, Reuss, and Hill EoS are consistent with the experimental results (Table 3). For the three EoS, the calculated Poisson ratios (Table 3) are also in excellent agreement with the experimental values. The Debye temperature $\left(\theta_{D}\right)$ of $\ln _{4} \mathrm{Se}_{3}$, which is related to the maximum phonon frequency $\left(\varpi_{D}=\frac{k_{B}}{\hbar} \theta_{D}\right)$, is low, $\sim 198 \mathrm{~K}$. This is also consistent with the low thermal conductivity this material exhibits. The phonon dispersion curves for $\ln _{4} \mathrm{Se}_{3}$ computed from first principles are presented in Figure 5. The absence of negative branches in the vibrational spectrum indicates that that the structure is thermodynamically stable. Therefore, a distortion leading to a superstructure is not expected for stoichiometric $\ln _{4} \mathrm{Se}_{3}$. This is entirely consistent with the structural study of Osters and coworkers ${ }^{33}$, who found no evidence of a Peierls-distortion or a CDW in stoichiometric $\ln _{4} \mathrm{Se}_{3}$. It is also noticeable that the frequency of the acoustic modes is very low, suggesting that the bonding is soft with a substantial number of low-frequency optical modes, close in energy to the acoustic modes. Although, per se, the vibrational spectrum is not sufficient to determine thermal transport quantities, the small energy difference between optical and acoustic modes suggests that the low-frequency optical phonon 
modes will interact strongly with the heat-carrying acoustic phonons, and may therefore be interpretative for the low thermal conductivity. By projecting the phonon density of states onto each atom, we find that the main contributors to low-frequency modes are the indium atoms, and in particular In4. This is consistent with the weak bonding we found for this atom in our structural analysis. Visualisations of the atom displacements for selected low-energy optical modes, together with the vibrational DOS resolved along different directions in the crystal structure, have been included as SI (Figure S5-S9). These indicate that the In4 atoms move mainly in the $a b$ plane. The large contribution of In4 to the eigendisplacement of the modes at low frequency is indicative of Einstein-like vibrations reminiscent of rattling. It is widely recognized that localized rattler modes within the acoustic range reduce the lattice thermal conductivity, either by resonant scattering or by a reduction in group velocity ${ }^{66}$. Given that our analysis of the bonding suggests the presence of In4 dimers, these rattling vibrations might involve pairs of In4 atoms. 
Table 3. Experimentally and computationally determined elastic properties of $\ln _{4} \mathrm{Se}_{3}$.

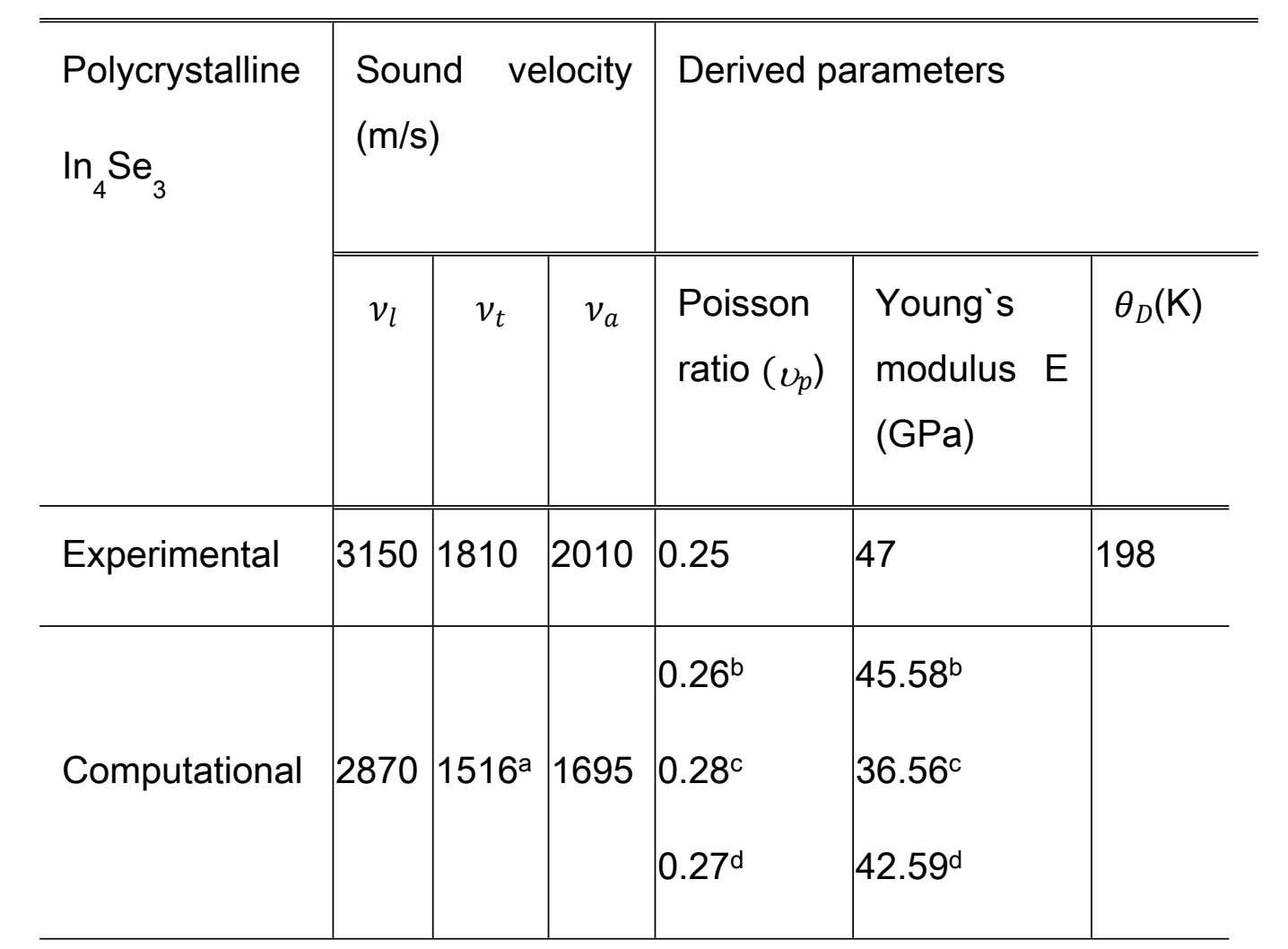

${ }^{a}$ Average transverse velocity; ${ }^{b}$ Voigt equation of state; ${ }^{c}$ Reuss equation of state; ${ }^{d}$ Hill equation of state

\section{Anharmonic Effects}

First-principles calculations within the quasi-harmonic approximation can be exploited to determine the mode-resolved Grüneisen parameter, which provides a direct measure of the 
anharmonicity of bonds (Figure 6(a)). We have demonstrated in the past ${ }^{67,68,69}$ that the presence of low-frequency anharmonic modes is a good descriptor for low thermal conductivity. Anharmonicity increases phonon-phonon scattering and therefore reduces the lattice thermal conductivity. As evidenced by Figure 6(a), the mode-resolved Grüneisen parameter for $\ln _{4} \mathrm{Se}_{3}$ is considerably larger for In atoms than for Se atoms. Moreover, the largest values of the Grüneisen parameter are found for In 4 between 20 and $50 \mathrm{~cm}^{-1}$. In the atom-projected vibrational density of states (Figure 5), this frequency range corresponds to the region where the Einsteinlike dispersion is observed. This is consistent with the weak bonding of In4 resulting in rattlinglike vibrations. Calculations of the total energy response to the in-plane displacement of In4 (Figure 6(b)) indicate that the total energy is minimally affected by displacements, and therefore confirm that the bonding of this atom is soft. It has been shown that anharmonicity can be amplified by lone-pair polarization ${ }^{70}$, which could be a contributive factor to the origin of the low thermal conductivity of $\ln _{4} \mathrm{Se}_{3}$, owing to the presence of a lone $5 s^{2}$ pair in $\ln 4$. Our structural analysis suggests that the In4 atoms, which exhibit a highly asymmetric bonding environment, might be forming weakly-interacting dimers (Figure 2). We conjecture that, during thermal 
vibrations, the interaction of the lone pairs along the $\ln 4 \ldots \ln 4$ direction will lead to high anharmonicity.

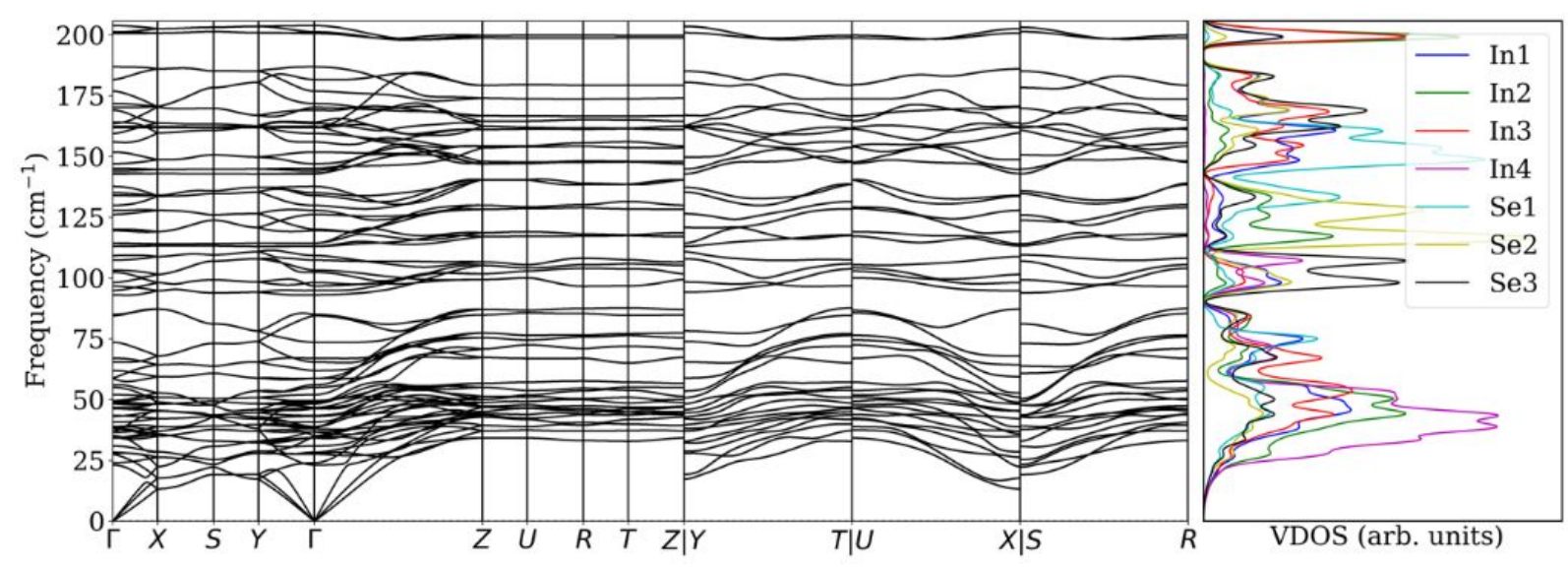

Figure 5. The computed phonons dispersion curves (left) for $\ln _{4} \mathrm{Se}_{3}$ from first-principles and atom-projected vibrational density of states (right). LO-TO splitting is very small. 
(a)

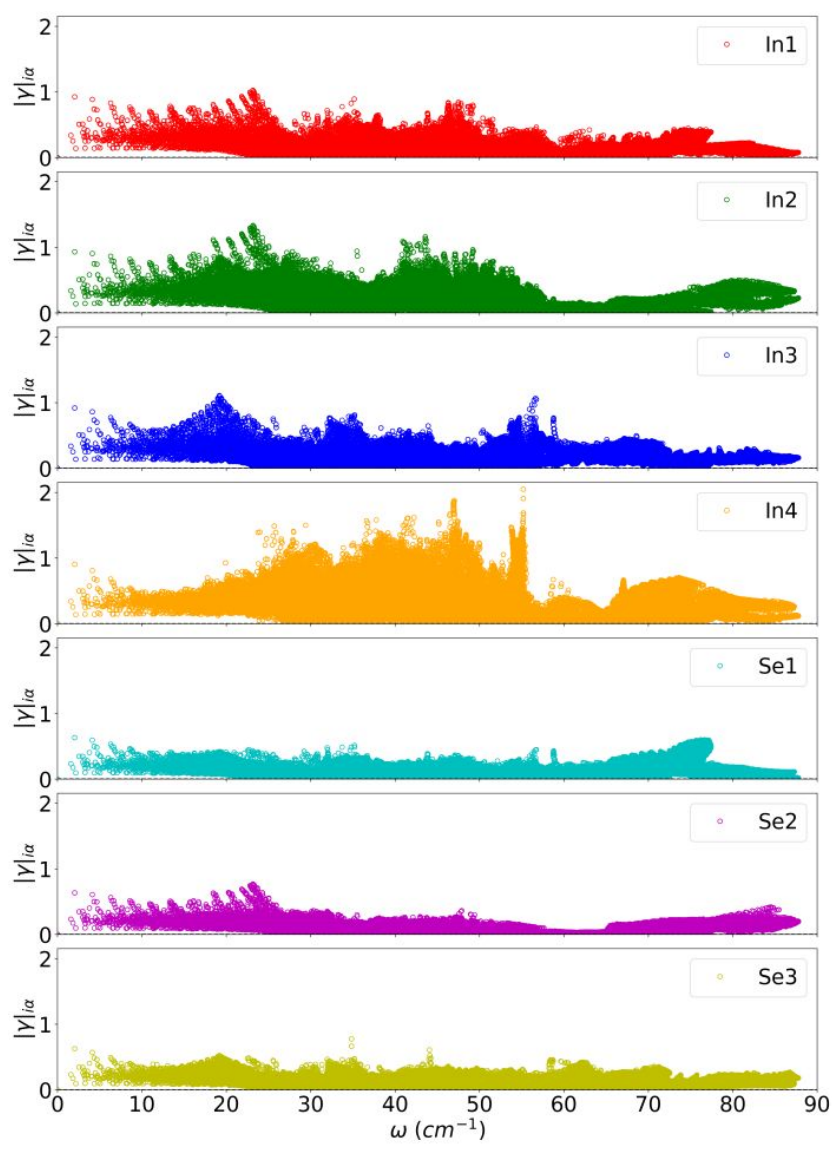

Figure 6. (a) Mode resolved Grüneisen parameters projected on individual atoms. (b) Total energy differences for the symmetrized displacement of the In4 atom along the [001] direction and in the plane $x-y$.

(b)

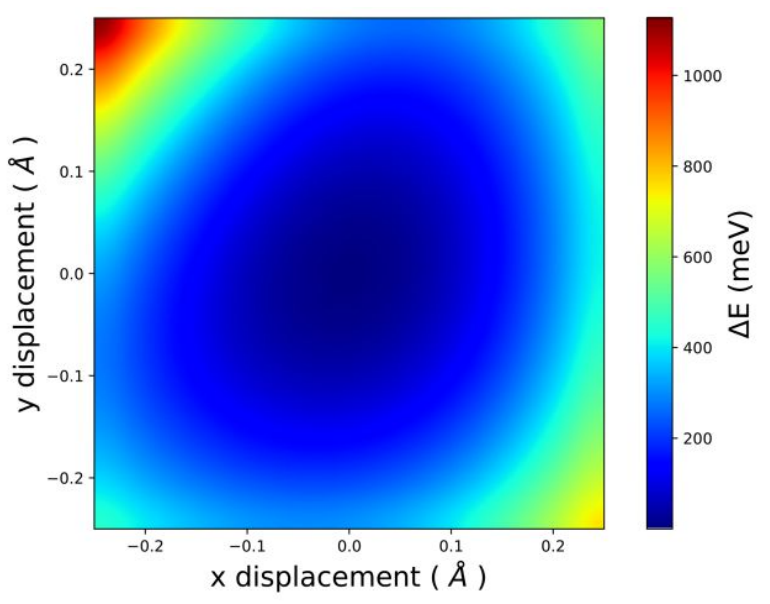




\section{CONCLUSIONS}

Our experimental and computational results demonstrate that, contrary to a previous suggestion which related low thermal conductivity to a Peierls distortion ${ }^{17}$, the intrinsically low thermal conductivity of $\ln _{4} \mathrm{Se}_{3}$ is a consequence of the soft bonding of $\mathrm{In}^{+}$ions located between covalently-bonded $\left[\left(\ln _{3}\right)^{5+}\left(\mathrm{Se}^{2-}\right)_{3}\right]^{-}$layers. This conclusion is strongly supported by the presence of Einstein-like modes in the vibrational density of states, which we attribute to "rattling" vibrations of the weakly-bonded $\mathrm{In}^{+}$cations. The synergistic effect of soft bonding and the lone $5 s^{2}$ pair of the $\mathrm{In}^{+}$cations leads to a high degree of anharmonicity, as evidenced by large moderesolved Grüneisen parameters, and hence to more effective phonon scattering.

\section{ACKNOWLEDGMENTS}

S. D. N. L and K.T.W thank the Institute of Advanced Manufacturing Technology for hosting and financial support within the TEAM -TECH0076 project of the Foundation for Polish Science, entitled "New approach for the development of efficient materials for direct conversion of heat into electricity project", co-financed by the European Union under the European Regional Development Fund. P.V. acknowledges The Leverhulme Trust for Research Project Grant 
(RPG-2019-288). A.R.S. and M.F. thank the Institute for Cyber-Enabled Research at Michigan

State University for access to computational facilities, and Giovanni Pizzi at materialscloud.org

for technical support.

\title{
Supporting Information
}

The following files are available free of charge:

Tables of bond lengths and angles, bond valence sums, powder XRD of powdered and pellet samples, SEM and EDS data, the calculated electronic structure, calculated Seebeck and electrical conductivity, the visualisations of the atom displacements, and vibrational DOS data (PDF).

\author{
AUTHOR INFORMATION \\ Corresponding Author \\ *E-mail: luudngocson@duytan.edu.vn; p.vaqueiro@reading.ac.uk
}

\section{Author Contribution}

The manuscript was written through the contributions of all authors. 


\section{Notes}

The authors declare that they have no known competing financial interests or personal relationships that could have appeared to influence the work reported in this paper. 


\section{REFERENCES}

(1) Rowe, D. M., Thermoelectrics Handbook: Macro to Nano, ed. D. M. Rowe, CRC Press, Taylor and Francis, Boca Raton, 2006, 1.

(2) Slack, G. A., New materials and performance limits for thermoelectric cooling. In $C R C$ Handbook of Thermoelectrics; Rowe. D.M., Ed.; CRC, Boca Raton, 1995, 34, 407-440.

(3) Yin,Y., Baskaran,K., Tiwari,A., A Review of Strategies for Developing Promising Thermoelectric Materials by Controlling Thermal Conduction, Phys. Status Solidi A, 2019, 1800904

(4) Yang, J., Wang, Y., Yang, H., Tang, W., Yang, J., Chen, L., Zhang, W., Thermal transport in thermoelectric materials with chemical bond hierarchy, J. Phys.: Condens. Matter, 2019, 31, 183002

(5) Liu, H., Shi, X., Xu, F., Zhang, L., Zhang, W., Chen, L., Li, Q., Uher, C., Day, T., Snyder, G.J., Copper ion liquid-like thermoelectrics, Nat. Mater., 2012, 11, 422-425. 
(6) Qiu, W., Xi, L., Wei, P., Ke, X., Yang,J., Zhang, W., Part-crystalline part-liquid state and rattling-like thermal damping in materials with chemical-bond hierarchy, Proc. Natl. Acad. Sci. USA $2014,111,15031$

(7) Li, D., Zhao, H., Li, Sh., Wei, B., Shuai, J., Shi,Ch., Xi, X., Sun, P., Meng, Sh., Gu, L., Ren,

Zh., Chen, X., Atomic Disorders Induced by Silver and Magnesium lon Migrations Favor High Thermoelectric Performance in a-MgAgSb Based Materials, Adv. Funct. Mater. 2015, 25, 64786488

(8) Zhao,K., Qiu,P., Shi,X., Chen, L., Recent Advances in Liquid-Like Thermoelectric Materials, Adv. Funct. Mater. 2019, 1903867

(9) Kim, S.II., Lee, K.H., Mun, H.A., Kim, H.S., Hwang, S.W., Roh, J.W., Yang, D.J., Shin, W.H., Li, X.S, Lee, Y.H., Snyder, G.J., Kim, S., Dense dislocation arrays embedded in grain boundaries for high-performance bulk thermoelectrics, Science, 2015, 348, 109-114.

(10) Kim,W., Strategies for engineering phonon transport in thermoelectrics, J. Mater. Chem.

C, 2015, 3, 10336-10348 
(11) Kanatzidis, M. G., Nanostructured thermoelectrics: The New Paradigm? Chem. Mater., 2010, 22, 648-659.

(12) Samanta, M., Pal, K., Pal, P., Waghmare, U. V., Biswas, K., Localized Vibrations of Bi Bilayer Leading to Ultralow Lattice Thermal Conductivity and High Thermoelectric Performance in Weak Topological Insulator n-Type BiSe, J. Am. Chem. Soc. 2018, 140, 17, 5866-5872.

(13) Acharyya, P., Ghosh, T., Pal, K., Kundu, K., Rana, K. S., Pandey, J., Soni, A., Waghmare, U. V., Biswas, K., Intrinsically Ultralow Thermal Conductivity in Ruddlesden-Popper 2D Perovskite $\mathrm{Cs}_{2} \mathrm{Pbl}_{2} \mathrm{Cl}_{2}$ : Localized Anharmonic Vibrations and Dynamic Octahedral Distortions, J. Am. Chem. Soc. 2020, 142, 36, 15595-15603.

(14) Sarkar, D., Ghosh, T., Roychowdhury, S., Arora, R., Sajan, S., Sheet, G., Waghmare, U. V., Biswas, K., Ferroelectric Instability Induced Ultralow Thermal Conductivity and High Thermoelectric Performance in Rhombohedral p-Type GeSe Crystal, J. Am. Chem. Soc. 2020, $142,28,12237-12244$. 
(15) Dutta, M., Matteppanavar, S., Prasad, M. V. D., Pandey, J., Warankar, A., Mandal, P.,

Soni, A., Waghmare, U. V., Biswas, K., Ultralow Thermal Conductivity in Chain-like TISe Due to Inherent TI+ Rattling, J. Am. Chem. Soc. 2019, 141, 51, 20293-20299.

(16) Rathore, E., Juneja, R., Culver, S. P., Minafra, N., Singh, A. K., Zeier, W. G., Biswas, K., Origin of Ultralow Thermal Conductivity in n-Type Cubic Bulk $\mathrm{AgBiS}_{2}$ : Soft Ag Vibrations and Local Structural Distortion Induced by the Bi $6 s^{2}$ Lone Pair, Chem. Mater. 2019, 31, 6, $2106-$ 2113.

(17) Rhyee, J. S., Lee, K.H., Lee, S. M., Cho, E., Kim, S. I., Lee, E., Kwon, Y. S., Shim, J. H., Kotliar, G., Peierls distortion as a route to high thermoelectric performance in $\ln _{4} \mathrm{Se}_{3-\delta}$ crystals, Nature , 2009, 459, 965.

(18) Rhyee, J. S., Ahn, K., K.H., Lee, Ji, H. S., Shim, J.H., Enhancement of the Thermoelectric Figure-of-Merit in a Wide Temperature Range in $\ln _{4} \mathrm{Se}_{3-x} \mathrm{Cl}_{0.03}$ Bulk Crystals, $A d v$. Mater., 2011, 23, 2191. 
(19) Lin, Z. S., Chen, L., Wang, L. M., Zhao, J. T., Wu, L. M., A Promising Mid-Temperature

Thermoelectric Material Candidate: $\mathrm{Pb} / \mathrm{Sn}$-Codoped $\operatorname{In}_{4} \mathrm{~Pb}_{\mathrm{x}} \mathrm{Sn}_{\mathrm{y}} \mathrm{Se}_{3}$ Adv. Mater., 2013, 25, 4800

-4806 .

(20) Han, G., Chen, Z-G., Drennan, J., Zou, J., Indium Selenides: Structural Characteristics, Synthesis and Their Thermoelectric Performances, Small, 2014, 14, 10, 2747-2765.

(21) Yin, X., Liu, J-Y., Chen, L., Wu, L-M., High Thermoelectric Performance of $\operatorname{In}_{4} \mathrm{Se}_{3}$-Based Materials and the Influencing Factors, Acc. Chem. Res., 2018, 51, 2, 240-247.

(22) Ahn, K., Cho, E., Rhyee, J. S., Kim , S. II., Lee , S. M., Lee , K. H., Effect of cationic substitution on the thermoelectric properties of $\ln _{4-x} \mathrm{M}_{\mathrm{x}} \mathrm{Se}_{2.95}$ compounds ( $\mathrm{M}=\mathrm{Na}, \mathrm{Ca}, \mathrm{Zn}, \mathrm{Ga}$, Sn, Pb; x=0.1), Appl. Phys. Lett., 2011, 99, 102110.

(23) Li, G., Yang, J. Y., Luo, Y. B., Xiao, Y., Fu, L. W., Liu, M., Peng, J. Y., Improvement of Thermoelectric Properties of $\operatorname{In}_{4} \mathrm{Se}_{3}$ Bulk Materials with Cu Nanoinclusions, J. Am. Ceram. Soc., 2013, 96, 2703. 
(24) J. Y., Luo, Yang, J. Y., Li, G., Liu, M., Xiao, Y., Fu, L. W., Li, W. X., Zhu, P. W., Peng, J. Y, Gao, S., Zhang, J. Q., Enhancement of the Thermoelectric Performance of Polycrystalline $\ln _{4} \mathrm{Se}_{2.5}$ by Copper Intercalation and Bromine Substitution, Adv. Energy Mater. 2014, 4, 1300599.

(25) Lee, M. H., Rhyee, J. S., Vaseem, M., Hahn, Y. B., Park, S. D., Kim, H. J., Kim, S. J., Lee, H. J., Kim, C., Thermoelectric properties of $\mathrm{SrTiO}_{3}$ nano-particles dispersed indium selenide bulk composites, Appl. Phys. Lett., 2013, 102 ,223901.

(26) Zhai, Y. B., Zhang, Q. S., Jiang, J., Zhang, T., Xiao, Y. K., Yang, S. H., Xu, G. J., Thermoelectric performance of the ordered $\ln _{4} \mathrm{Se}_{3}-\mathrm{In}$ composite constructed by monotectic solidification, J. Mater. Chem. A, 2013, 1, 8844-8847.

(27) Rawat, P. K., Park, H., Hwang, J., Kim, W., Low Thermal Conductivity and High Thermoelectric Performance in $\ln _{4} \mathrm{Se}_{3-\mathrm{x}}$ with Phase-Separated Indium Inclusions, J. Elec. Mater. 2017, 46, 1444-1450. 
(28) Zhu, G.H., Lan, Y.C., Wang, H., Joshi, G., Hao, Q., Chen, G., Ren, Z.F. , Effect of selenium deficiency on the thermoelectric properties of n-type $\ln _{4} S_{3-x}$ compounds, Phys. Rev. B, 2011, 83, 115201.

(29) Ahn, K., Cho, E., Rhyee, J. S., Kim, S. II., Hwang, S., Kim, H-S., Lee, S. M., Lee, K. H., Improvement in the thermoelectric performance of the crystals of halogen-substituted $\mathrm{In}_{4} \mathrm{Se}_{3-\mathrm{x}} \mathrm{H}_{0.03}(\mathrm{H}=\mathrm{F}, \mathrm{Cl}, \mathrm{Br}, \mathrm{I})$ : Effect of halogen-substitution on the thermoelectric properties in $\ln _{4} \mathrm{Se}_{3-x}$ J. Mater. Chem., 2012, 22, 5730-5736.

(30) Kim, J. H., Kim, M. J., Oh, S., Rhyee, J-S., Thermoelectric properties of Se-deficient and $\mathrm{Pb}-/ \mathrm{Sn}$-codoped $\mathrm{In}_{4} \mathrm{~Pb}_{0.01} \mathrm{Sn}_{0.03} \mathrm{Se}_{3-x}$ polycrystalline compounds, J. Alloy. Compd., 2014, 615, 933-936.

(31) Abharia, A. S., Abdellahib, M., Bahmanpour, M., The effects of Sn-substitution on thermoelectric properties of $\ln _{4-x} \mathrm{Sn}_{x} \mathrm{Se}_{3}$ ceramic, Ceram. Int., 2016, 42, 5593-5599. 
(32) Luu, S. D. N., Parashchuk, T., Kosonowski, A., Phan, T. B., Wojciechowski, K. T., Structural and Thermoelectric Properties of Solid-Liquid $\operatorname{In}_{4} \mathrm{Se}_{3}$-In Composite, J. Elec. Mater. $2019,48,5418-5427$.

(33) Osters, O., Blazek, G., Nilges, T., Comments on Peierls-distorted Indium Chains in $\ln _{4} \mathrm{Se}_{3-}$ x, Z. Anorg. Allg. Chem. 2013, 639, 497-501.

(34) Jia, T., Chen, G., Zhang, Y., Lattice thermal conductivity evaluated using elastic properties, Phys. Rev. B, 2017, 95, 155206.

(35) Ji, H. S., Kim, H., Lee, C., Rhyee, J-S., Kim, M. H., Kaviany, M., Shim, J. H., Vacancysuppressed lattice conductivity of high-ZT $\ln _{4} \mathrm{Se}_{3-\mathrm{x}}$, Phys. Rev. B, 2013, 87, 125111.

(36) Kuryachii, V. Y., Bogachev, V. Y., Mikhal'chenko, V. P., Stakhira, I. M., Elastic properties of $\mathrm{In}_{4} \mathrm{Se}_{3}$, Izv. Akad. Nauk SSSR, Neorg. Mater. 1986, 18, 756-757.

(37). Zevalkink, A.; Smiadak, D. M.; Blackburn, J. L.; Ferguson, A. J.; Chabinyc, M. L.; Delaire, O.; Wang, J.; Kovnir, K.; Martin, J.; Schelhas, L. T.; Sparks, T. D.; Kang, S. D.; Dylla, M. T.; 
Snyder, G. J.; Ortiz, B. R.; Toberer, E. S. A practical field guide to thermoelectrics:

Fundamentals, synthesis, and

characterization, App. Phys. Rev. 2018, 5, 021303.

(38) Asmani, M., Kermel, C., Leriche, A., Ourak, M., Influence of porosity on Young's modulus and Poisson's ratio in alumina ceramics, J. Eur. Ceram. Soc., 2001, 21, 1081-1086.

(39) Anderson, O. L., A simplified method for calculating the Debye temperature from elastic constants, J. Phys. Chem. Solids, 1963, 24, 909-917.

(40) Kurosaki, K., Kosuga, A., Muta, H., Uno, M., Yamanaka, S., Ag9 $\mathrm{TITe}_{5}$ : A high-performance thermoelectric bulk material with extremely low thermal conductivity, Appl. Phys. Lett., 2005, 87, 061919.

(41) Sanditov, D. S., Belomestnykh, V. N., Relation between the parameters of the elasticity theory and averaged bulk modulus of solids, Tech. Phys. 2011, 56, 1619-1623. 
(42) Belomestnykh, V.N., Tesleva, E.P., Interrelation between anharmonicity and lateral strain in quasi-isotropic polycrystalline solids, Tech. Phys. 2004, 49, 1098-1100.

(43) Kim, H.-S.; Gibbs, Z. M.; Tang, Y.; Wang, H.; Snyder, G. J. APL Materials, 2015, 3, 041506.

(44) Slack, G., Nonmetallic crystals with high thermal conductivity, J. Phys. Chem. Solids, 1973, 34, 321-335.

(45) Cahill, D. G., Watson, S. K., Pohl, R. O., Lower limit to the thermal conductivity of disordered crystals, Phys. Rev. B 1992, 46, 6131-6140

(46) Giannozzi, P., Baroni, S., Bonini, N., Calandra, M., Car, R., Cavazzoni, C., Ceresoli, D., Chiarotti, G. L, Cococcioni, M., Dabo, I., Corso, A. Dal., Gironcoli, S. de, Fabris, S., Fratesi, G., Gebauer, R., Gerstmann, U., Gougoussis, C., Kokalj, A., Lazzeri, M., Martin-Samos, L., Marzari, N., Mauri, F., Mazzarello, R., Paolini, S., Pasquarello, A., Paulatto, L., Sbraccia, C., Scandolo, S., Sclauzero, G., Seitsonen, A. P., Smogunov, A., Umari, P., Wentzcovitch, R. M., QUANTUM 
ESPRESSO: a modular and open-source software project for quantum simulations of materials, J. Phys.: Condens. Matter, 2009, 21, 395502

(47) Supka, A. R., Lyons, T. E., Liyanage, L., D’Amico, P., Al Rahal Al Orabi, R., Mahatara, S., Gopal, P., Toher, C., Ceresoli, D., Calzolari, A., Curtarolo, S., Nardelli, M. B., Fornari, M., AFLOWm: A minimalist approach to high-throughput ab initio calculations including the generation of tight-binding hamiltonians, Comput. Mater. Sci., 2017, 136, 76-84.

(48) Setten, M. J. V., Giantomassi, M., Bousquet, E., Verstraete, M. J., Hamann, D. R., Gonze, X., Rignanese, G.-M., The PseudoDojo: Training and grading a 85 element optimized normconserving pseudopotential table, Comput. Phys. Commun, 2018, 226, 39-54.

(49) Nardelli, M. B., Cerasoli, F. T., Costa, M., Curtarolo, S., De Gennaro, R., Fornari, M., Liyanage, L., Supka, A. R., Wang, H., PAOFLOW: A utility to construct and operate on ab initio Hamiltonians from the projections of electronic wavefunctions on atomic orbital bases, including characterization of topological materials, Comp. Mat. Sci. 2018, 143, 462-472 
(50) Golesorkhtabar, R., Pavone, P., Spitaler, J., Puschnig, P., Draxl, C., ElaStic: A tool for calculating second-order elastic constants from first principles, Comput. Phys. Commun, 2013, $184,1861-1873$

(51) Zhang, Y., First-principles Debye-Callaway approach to lattice thermal conductivity, J.

Materiomics, 2016, 2, 237-247

(52) Hogg, J. H. C., Sutherland, H. H., Williams, D. J., The crystal structure of tetraindium triselenide, Acta Crystallogr. B, 1973, B29, 1590-1593

(53) Wolcyrz, M., Kubiak, R., Maciejewski, S., X-ray investigation of thermal expansion and atomic thermal vibrations of tin, indium, and their alloys, Phys. Status Solidi B, 1981, 107, 245253

(54) Cordero, B., Gómez, V., Platero-Prats, A. E., Revés, M., J. Echeverría, E. Cremades, F.Barragán, S. Alvarez, Covalent radii revisited, Dalton Trans., 2008, 21, 2832-2838 
(55) Jones, R. E., Templeton, D. H., The crystal structure of indium (I) iodide, Acta Crystallogr.

$1955,8,847$

(56) Shannon, R. D., Revised effective ionic radii and systematic studies of interatomic distances in halides and chalcogenides, Acta Crystallogr A. 1976, A32: 751-767

(57) Losovyj, Y. B., Makinistian, L., Albanesi, E. A., Petukhov, A. G., Liu, J., Galiy, P., Dveriy, O. R., Dowben, P. A., The anisotropic band structure of layered In4Se3(001), J. Appl. Phys., $2008,104,083713$.

(58) Walsh, A., Payne, D. J., Egdell, R. G., Watson, G. W., Stereochemistry of post-transition metal oxides: revision of the classical lone pair model, Chem. Soc. Rev., 2011, 40, 4455-4463

(59) Hanus, R., Agne, M. T., Rettie, A. J. E., Chen, Z., Tan, G., Chung, D. Y., Kanatzidis, M.G., Pei, Y., Voorhees, P. W., Snyder, G. J., Lattice Softening Significantly Reduces Thermal Conductivity and Leads to High Thermoelectric Efficiency, Adv. Mater. 2019, 31, 1900108 
(60) Kallel, A.C., Roux, G., Martin, C.L., Thermoelectric and mechanical properties of a hot pressed nanostructured n-type $\mathrm{Si}_{80} \mathrm{Ge}_{20}$ alloy, Mater. Sci. Eng. A., 2013, 564, 65-70

(61) Schmidt, R., Fan, X., Case, E., Sarac, P., Mechanical properties of $\mathrm{Mg}_{2}$ Si thermoelectric materials with the addition of $0-4$ vol\% silicon carbide nanoparticles (SiCNP), J. Mater. Sci., $2015,50,11,4034-4046$

(62) Ni, E. J., Casea, D.E., Khabir, N.K., Stewart, C.R., Wub, Ch-I., Hoganb, P.T., Timmc, J. E., Girardd, N.S., Kanatzidis, G. M., Room temperature Young's modulus, shear modulus, Poisson's ratio and hardness of PbTe-PbS thermoelectric materials, Mat. Sci. Eng. B, 2010, $170,58-66$

(63) Li, M. Kazi Nazrul Islam, Md. Sh., Yahyaoglu,M., Pan,D., Shi,X., Chen, L.D., Aydemir. U., Wang, X., Ultrahigh figure-of-merit of $\mathrm{Cu}_{2} \mathrm{Se}$ incorporated with carbon coated boron nanoparticles, InfoMat. 2019;1:108-115 
(64) Ashby, M. F., Materials Selection in Mechanical Design, $4^{\text {th }}$ Edi. Butterworth Heinemann, 2011

(65) Lin, S., Li, W., Li, S., Zhang, X., Chen, Z., Xu, Y., Chen, Y., Pei, Y., High Thermoelectric Performance of $\mathrm{Ag}_{9} \mathrm{GaSe}_{6}$ Enabled by Low Cut off Frequency of Acoustic Phonons, Joule, 2017, $1,816-830$

(66) Toberer, E.S., Zevalkink, A., Snyder, G.J., Phonon engineering through crystal chemistry, J. Mater. Chem., 2011, 21, 15843-15852

(67) Vaqueiro, P., Al Orabi, R. A. R., Luu, S. D. N., Guélou, G., Powell, A. V., Smith, R. I., Song, J.-P., Wee, D., Fornari, M., The Role of Copper in The Thermal Conductivity of Thermoelectric Oxychalcogenides: Do Lone Pairs Matter?, Phys. Chem. Chem. Phys. 2015, 17, 31735- 31740.

(68) Plata, J. J., Nath, P., Usanmaz, D., Carrete, J., Toher, C., Jong, M. D., Asta, M. D., Fornari, M., Nardelli, M. B., Curtarolo, S., An efficient and accurate framework for calculating lattice 
thermal conductivity of solids: AAPL - AFLOW Anharmonic Automatic Phonon Library, NPJ Comp. Mat. 2017, 3, 45 .

(69) Bourgs, C., Bouyrie, Y., Supka, A., Al Orabi, R. A. R, Lemoine, P., Lebedev, O., Ohta, M., Suekuni, K., Nassif, V., Hardy, V., Daou, R., Miyazaki, Y., Fornari, M., Guilmeau, E., Highperformance Thermoelectric Bulk Colusite by Process Controlled Structural Disordering, J. Am. Chem. Soc. 2018, 140, 2186-2195 .

(70) Nielsen, M. D., Ozolins, V., Heremans, J. P., Lone pair electrons minimise lattice thermal conductivity, Energy Environ. Sci. 2013, 6, 570-578. 


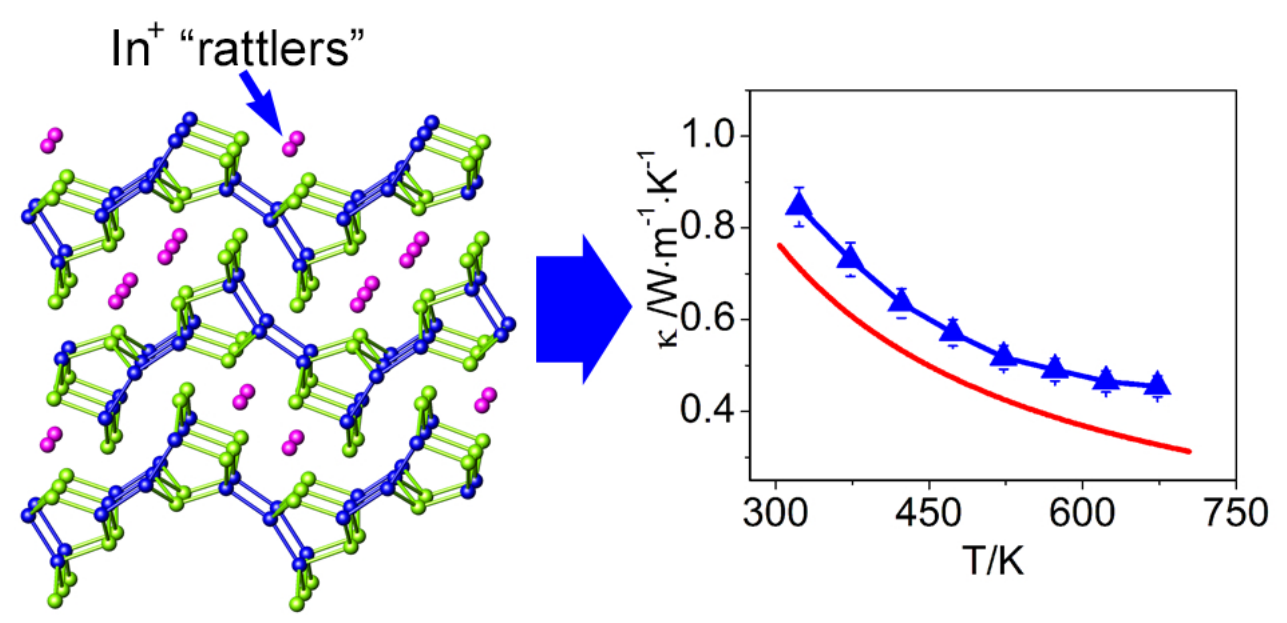

$82 \times 44 \mathrm{~mm}(300 \times 300$ DPI $)$ 